\title{
Preparative Steps Towards the New Definition of the Kelvin in Terms of the Boltzmann Constant
}

\author{
J. Fischer • S. Gerasimov • K. D. Hill • G. Machin • \\ M. R. Moldover • L. Pitre - P. Steur • M. Stock • O. Tamura • \\ H. Ugur • D. R. White • I. Yang • J. Zhang
}

Published online: 15 November 2007

(C) Springer Science+Business Media, LLC 2007

\begin{abstract}
The International Committee for Weights and Measures (CIPM) approved, in its Recommendation 1 of 2005, preparative steps towards new definitions of the kilogram, the ampere, the kelvin, and the mole in terms of fundamental constants. Within the Consultative Committee for Thermometry (CCT), a task group (TG-SI)
\end{abstract}

H. Ugur is the President, Consultative Committee for Thermometry (CCT).

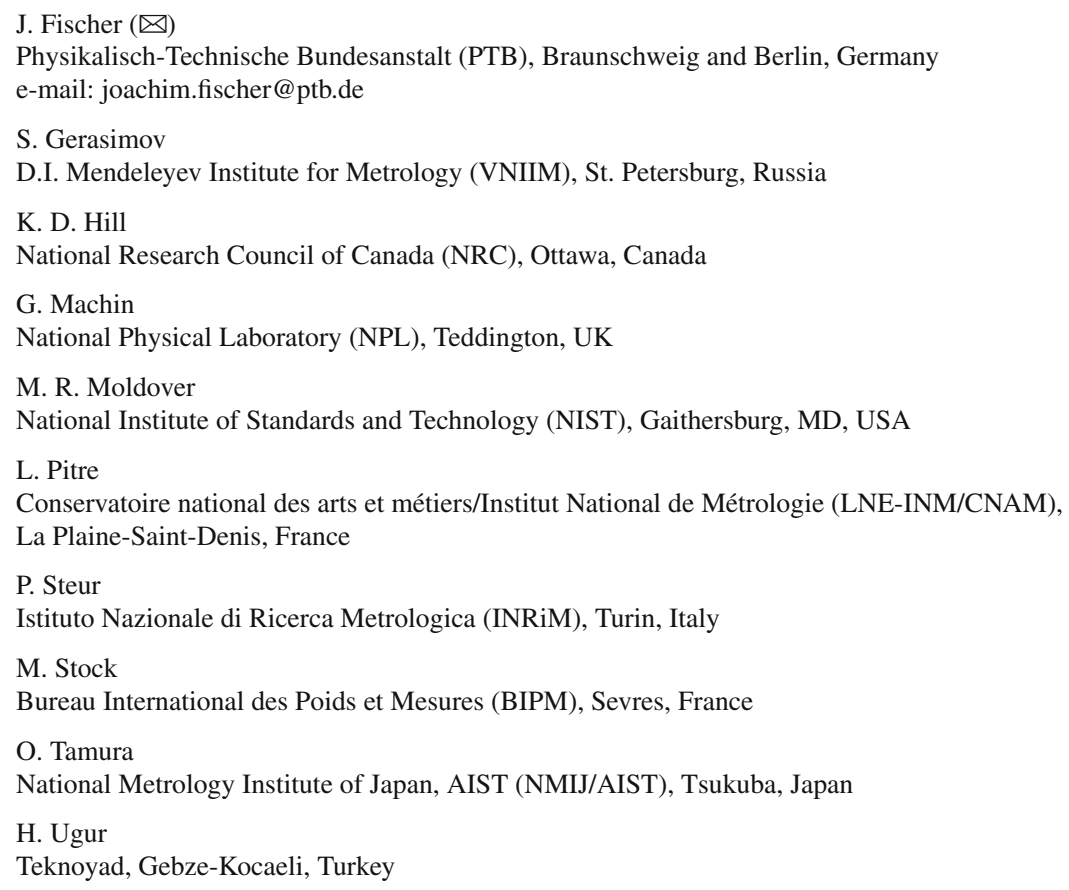


has been formed to consider the implications of changing the definitions of the above-mentioned base units of the SI, with particular emphasis on the kelvin and the impact of the changes on metrology in thermometry. The TG-SI has presented the results of its deliberations to the CCT and to the Consultative Committee for Units, $\mathrm{CCU}$, and worked with them to prepare a report to the CIPM. This contribution, authored by the members of TG-SI, solicits input from the wider scientific and technical community on this important matter at the TEMPMEKO 2007 conference. For this purpose, the main details of the report to the CIPM are presented. The unit of temperature $T$, the kelvin, can be defined in terms of the SI unit of energy, the joule, by fixing the value of the Boltzmann constant $k$, which is simply the proportionality constant between temperature and thermal energy $k T$. Currently, several experiments are underway to determine $k$. The TG-SI is monitoring closely the results of all experiments relevant to the possible new definition of the kelvin, and has identified conditions to be met before proceeding with the proposed redefinition. The TG-SI considers that these conditions will be fulfilled before the 24th General Conference on Weights and Measures in October 2011. Therefore, the TG-SI is recommending a redefinition of the kelvin by fixing the value of the Boltzmann constant. A new definition of the kelvin in terms of the Boltzmann constant does not require the replacement of ITS-90 with an improved temperature scale nor does it prevent such a replacement.

Keywords Boltzmann constant - Fundamental physical constants - International System of Units · Kelvin · Primary thermometry

\section{Introduction}

Thermometers that can be used to determine thermodynamic temperature directly are few in number, difficult to employ, expensive, and not as precise or reproducible as many practical thermometers. To meet the need for practical temperature measurement, International Temperature Scales have been defined (ITS-27, IPTS-48, IPTS-68, ITS90, named after the year of promulgation) [1], which are essentially recipes for the realization of highly reproducible and precise temperature standards which are in close accord with the best thermodynamic measurements of the time. These scales have been based on sets of fixed points, the defined temperatures of equilibrium states of certain specified pure substances, and specified methods for interpolating or extrapolating from these points [2].

Thus, the quantity determined in the vast majority of present-day temperature measurements is not thermodynamic temperature but $T_{90}$, as defined by the International Temperature Scale of 1990, ITS-90 [3]. ITS-90 covers the range from $0.65 \mathrm{~K}$ to the

\footnotetext{
D. R. White

Measurement Standards Laboratory of New Zealand (MSL), Lower Hutt, New Zealand

I. Yang

Korea Research Institute of Standards and Science (KRISS), Daejeon, Korea

J. Zhang

National Institute of Metrology (NIM), Beijing, P.R. China
} 
highest temperature measurable in practice using the Planck radiation law. The ITS90 has recently been supplemented by the Provisional Low Temperature Scale from $0.9 \mathrm{mK}$ to $1 \mathrm{~K}$ (PLTS-2000) [4], which covers the range from $0.9 \mathrm{mK}$ to $1 \mathrm{~K}$ and defines the quantity $T_{2000}$.

Recent developments in thermodynamic thermometry [5] have, for the first time, offered primary thermometers with an accuracy which can approach or exceed the precision of ITS-90, and with sufficient convenience to employ as standards. In principle, this makes it possible, for some temperature ranges at least, to dispense with ITS-90 and measure true thermodynamic temperatures.

For many years, the CIPM has had the long-term aim of defining all of the base units in terms of fundamental physical constants to eliminate any artifact or material dependences and ensure the long-term stability of the units. In its Recommendation 1 of 2005, the CIPM approved preparative steps towards new definitions of the kilogram, the ampere, the kelvin, and the mole in terms of fundamental constants [6]. Within the CCT, the task group TG-SI considered the implications of changing the definitions of the above-mentioned base units of the SI, with particular emphasis on the kelvin.

For the kelvin, the change would generalize the definition, making it independent of any material substance, technique of realization, and temperature or temperature range. In particular, the new definition would improve temperature measurement at temperatures far away from the triple point of water. For example, in the high temperature range, the radiometry community could apply absolute radiation thermometers without the need to refer to the triple point of water. It would also encourage the use of direct realizations of thermodynamic temperatures in parallel with the realization described in the International Temperature Scale. A new definition of the kelvin in terms of the Boltzmann constant does not require the replacement of ITS-90 with an improved temperature scale, nor does it prevent such a replacement. In the long term, it will enable gradual improvements to the temperature scale in respect of lower uncertainties and extended temperature ranges, without the high transitional costs and inconvenience that have been incurred with previous changes in temperature scales.

This paper, authored by the members of TG-SI, provides background information on the proposal and solicits input from the wider scientific and technical community on this important matter at the TEMPMEKO 2007 conference.

\section{Historical Background}

The current definition of temperature was first suggested by William Thomson, later Lord Kelvin, and is based on the amounts of heat entering and leaving an ideal heat engine,

$$
\frac{Q_{\mathrm{h}}}{T_{\mathrm{h}}}=\frac{Q_{\mathrm{c}}}{T_{\mathrm{c}}}
$$


where $Q_{\mathrm{h}}$ is the heat flowing into the engine from a hot reservoir at temperature $T_{\mathrm{h}}$, and $Q_{\mathrm{c}}$ is the heat flowing out of the engine to a cold reservoir at temperature $T_{\mathrm{c}}$. Clausius observed that one consequence of Thomson's definition is that the sum of all of the heats flowing out of a heat engine multiplied by $1 / T$ is identically zero for reversible processes and greater than zero for irreversible processes, i.e.,

$$
\sum_{i} \frac{Q_{i}}{T_{i}} \geq 0
$$

Clausius called the new quantity, $S=Q / T$, entropy, being a sort of transformed energy (from the Greek trope meaning transformation).

The temperature defined by Thomson was based on the conceptual device of the ideal heat engine; however, such a device is not necessary. The mathematician Carathéodory showed that, in any system, there exists a unique state variable that characterizes the reversibility of a process, and this variable is proportional to the integral of the heats associated with the process divided by a unique "integrating factor" [7]. Comparison of Carathéodory's result with Eq. 2 shows that the state variable is the entropy defined by Clausius and the integrating factor is the temperature defined by Thomson. Carathéodory's result leads to a definition of temperature:

$$
\frac{1}{T}=\frac{\mathrm{d} S}{\mathrm{~d} U}
$$

where $U$ is the internal energy of the system and $S$ is the conventional entropy.

Boltzmann subsequently showed that the entropy of a system is related to the number of ways the constituent atoms and molecules can be arranged into the observed macroscopic state:

$$
S=k \sigma=k \ln P
$$

where $k$ is a constant, $P$ is the probability of the system being in the observed state, and $\sigma$ is the entropy according to Boltzmann's statistical definition. In his original work, Boltzmann deduced the relation $\sigma=\ln P$, without the factor $k$. This is in line with the definition of entropy according to Shannon's information theory [8], and leads naturally to a thermodynamic temperature, $\tau$, measured in energy units (joule), $1 / \tau=\mathrm{d} \sigma / \mathrm{d} U$, so, in principle, we do not need a separate base unit for temperature, the kelvin. However, such a temperature scale would have impracticably small $\left(\sim 10^{-20}\right)$ and unfamiliar values. For this reason, history went a different way and Planck introduced the constant $k$, later named after Boltzmann, to provide the link to conventional definitions of entropy and thermodynamic temperature [9].

Equation 3 applied to a variety of idealized systems yields thermodynamic relations that can be used to measure temperature. For example, for an ideal gas, we can derive the equation of state,

$$
p V_{\mathrm{m}}=N_{\mathrm{A}} k T
$$


where $p$ is the pressure, $V_{\mathrm{m}}$ is the molar volume, and $N_{\mathrm{A}}$ is Avogadro constant, the number of particles per mole. Systems with equations of state that can be used to measure temperature are often called primary thermometers (see Sect. 4 for further examples).

The quantity $k T=\tau$, which occurs in the equations of state, is a characteristic energy determining the energy distribution among the particles of the system when it is in thermal equilibrium. Thus, for unbound atoms, temperature is proportional to the mean translational kinetic energy. Thermodynamic temperature is linear and rational: equal intervals or ratios of temperature correspond to equal differences or ratios of mean kinetic energy, and a single definition is required to fix the magnitude of the temperature unit. All other temperature values must then be determined by experiment, using a suitable thermal system and equation of state.

Today, the kelvin is defined in terms of the temperature of the triple point of water and the Boltzmann constant $k$ is a measured quantity. The CIPM proposal is to define a numerical value for $k$, from which it follows that all temperatures, including the triple point of water, must be measured. Of course, the adopted value for $k$ will be such that the temperature values will, as far as possible, remain unchanged.

\section{Implications of the New Definition of the Kelvin}

If the CIPM proposal is adopted, there will be a number of consequences for temperature measurement practice and the thermometry community. The most immediate impact of the change is that it will endorse and encourage the use of both thermodynamic and ITS-90 temperatures. The change to a numerical definition of $k$ will also impact uncertainties in thermodynamic temperature measurements. In addressing the consequences, the overriding factor is the need to minimize the transitional cost and inconvenience to the measurement community, while gaining the benefits of improved primary thermometry. This section summarizes some of the issues and how they may be addressed.

\subsection{Status of ITS-90}

It is expected that the new definition for the kelvin will have little immediate impact on the status of ITS-90. However, the ITS-90 will no longer be the only practical option for temperature measurement. Thus, the most immediate and beneficial consequence of the change is for temperatures below $\sim 20 \mathrm{~K}$ and above $\sim 1300 \mathrm{~K}$ where primary thermometers may offer users a lower thermodynamic uncertainty than is currently available with ITS-90. However, the ITS-90 will remain in use for the foreseeable future as a precise, reproducible, and convenient approximation to thermodynamic temperature.

The long-term consequence of the change is that, as the primary methods evolve and achieve lower uncertainties, they will become more widely used and will gradually replace ITS-90 as the basis of temperature measurement. As now, there will be a need to indicate whether the measurements and uncertainties refer to $T$ or $T_{90}$. 
Table 1 Defining fixed points of the ITS-90 with uncertainties $u\left(T_{90}\right)$ of the best practical realization in terms of ITS-90 and uncertainties $u(T)$ of the thermodynamic temperature

\begin{tabular}{llccc}
\hline 1 & 2 & 3 & 4 & 5 \\
Fixed point & $T_{90}(\mathrm{~K})$ & $u\left(T_{90}\right)(\mathrm{mK})$ & $u(T)(\mathrm{mK})$ & $u\left(T_{k \text { fixed }}\right)(\mathrm{mK})$ \\
\hline $\mathrm{Cu}$ & 1357.77 & 15 & 60 & 60.1 \\
$\mathrm{Au}$ & 1337.33 & 10 & 50 & 50.1 \\
$\mathrm{Ag}$ & 1234.93 & 1 & 40 & 40.1 \\
$\mathrm{Al}$ & 933.473 & 0.3 & 25 & 25.1 \\
$\mathrm{Zn}$ & 692.677 & 0.1 & 13 & 13.1 \\
$\mathrm{Sn}$ & 505.078 & 0.1 & 5 & 5.10 \\
$\mathrm{In}$ & 429.7485 & 0.1 & 3 & 3.11 \\
$\mathrm{Ga}$ & 302.9146 & 0.05 & 1 & 1.15 \\
$\mathrm{H}_{2} \mathrm{O}$ & 273.16 & 0.02 & 0 & 0.49 \\
$\mathrm{Hg}$ & 234.3156 & 0.05 & 1.5 & 1.55 \\
$\mathrm{Ar}$ & 83.8058 & 0.1 & 1.5 & 1.50 \\
$\mathrm{O}_{2}$ & 54.3584 & 0.1 & 1 & 1.00 \\
$\mathrm{Ne}$ & 24.5561 & 0.2 & 0.5 & 0.50 \\
$\mathrm{e}-\mathrm{H}_{2}$ & $\approx 20.3$ & 0.2 & 0.5 & 0.50 \\
$\mathrm{e}-\mathrm{H}_{2}$ & $\approx 17.0$ & 0.2 & 0.5 & 0.50 \\
$\mathrm{e}-\mathrm{H}_{2}$ & 13.8033 & 0.1 & 0.5 & 0.50 \\
$4{ }_{\mathrm{He}}$ & 4.2221 & 0.1 & 0.3 & 0.30 \\
\hline$u\left(T_{k}\right.$ & & & & \\
\hline
\end{tabular}

$u\left(T_{k \text { fixed }}\right)$ is the uncertainty in the thermodynamic temperature of the listed phase transitions (which presently serve as fixed points on ITS-90) assuming a new definition for the kelvin is adopted with a fixed value for the Boltzmann constant. All values are quoted as standard uncertainties. Values in columns 3 and 4 have been taken from Table 1.2 of the Supplementary Information for the ITS-90 [2]

For the foreseeable future, most temperature measurements in the core temperature range from about -200 to $960^{\circ} \mathrm{C}$ will continue to be made using standard platinum resistance thermometers calibrated according to ITS-90. Because ITS-90 will remain intact, with defined values of $T_{90}$ for all of the fixed points, the uncertainties in $T_{90}$ will not change: they will continue to be dominated by uncertainties in the fixed-point realizations (column 3 of Table 1) and the non-uniqueness of the platinum resistance thermometers, typically totalling less than $1 \mathrm{mK}[10]$.

\subsection{Uncertainties in Thermodynamic Temperatures}

If the 2002 CODATA recommended value [11] of $k=1.3806505 \times 10^{-23} \mathrm{~J} \cdot \mathrm{K}^{-1}$ were taken to be exact and used to define the kelvin, the relative uncertainty in $k$, currently $1.8 \times 10^{-6}$, would be transferred to the temperature of the triple-point of water, $T_{\mathrm{TPW}}$. This means that if such a new definition were to be adopted today, our best estimate of the value of $T_{\mathrm{TPW}}$ would still be $273.16 \mathrm{~K}$, but instead of this value being exact as a result of the definition of the kelvin as is now the case, the standard uncertainty of the $T_{\mathrm{TPW}}$ would be $u\left(T_{\mathrm{TPW}}\right)=0.49 \mathrm{mK}$. 
Because all thermodynamic measurements are currently defined as ratios with respect to the triple point of water, the $0.49 \mathrm{mK}$ uncertainty propagates to all historical thermodynamic temperature measurements. In practice, the change in definition will only affect measurements made close to $273 \mathrm{~K}$ because the uncertainties of the thermodynamic temperatures well away from this are very much larger than $0.49 \mathrm{mK}$. To illustrate this point, columns 4 and 5 of Table 1 show for the defining fixed points of ITS-90 the current [2] $u(T)$ values and the uncertainties when the value of the Boltzmann constant will have been fixed.

The TG-SI could not foresee any experiment where the slightly increased uncertainties of thermodynamic temperatures $u\left(T_{k}\right.$ fixed $)$ would present a problem to metrology or the wider research community. It is also expected that any future changes in the temperature scale will be much smaller than the tolerances associated with current documentary standards for thermocouples [12] and industrial platinum resistance thermometers [13]. Therefore, no requirement is anticipated for any future change in temperature scales to propagate to the documentary standards. Once the Boltzmann constant has been fixed, which is expected to occur in 2011, the TG-SI is not aware of any new technology for a primary thermometer providing a significantly improved uncertainty $u\left(T_{\mathrm{TPW}}\right)$. Consequently, there will be no change of the assigned value of $T_{\mathrm{TPW}}$ for the foreseeable future.

The triple point of water will continue to have a role in practical thermometry. In fact, the inconsistency of $T_{\mathrm{TPW}}$ as realized by different triple-point-of-water reference cells can be as small as $50 \mu \mathrm{K}$, or even smaller, if the isotopic composition of the water used is taken into account $[14,15]$. Consequently, long-term experiments requiring ultimate accuracy at or close to $T_{\mathrm{TPW}}$ will still rely on the reproducibility of the triple point of water. To overcome this situation, a determination of the thermodynamic temperature $T_{\mathrm{TPW}}$ would be required with an uncertainty smaller than about $50 \mu \mathrm{K}$. This corresponds to a relative uncertainty in temperature of $2 \times 10^{-7}$.

\subsection{The Mise en Pratique}

To help users make accurate and reliable temperature measurements, the CIPM, through its Consultative Committee on Thermometry (CCT) and the BIPM, is publishing a collection of guidelines for temperature measurement. This is similar to the current Supplementary Information for the International Temperature Scale of 1990, also published by the BIPM [2]. Following the practice established for length measurements, the guidelines are referred to as the mise en pratique of the definition of the Kelvin (MeP) [16] and comprise recognized approximations to thermodynamic temperature currently including ITS-90 and PLTS-2000. The MeP will, in the future, be expanded to describe recognized primary methods for measuring temperature or realizing the scale, and the sources of uncertainty associated with the measurements. The $\mathrm{MeP}$ will be updated regularly as primary methods improve. Although there will be no immediate changes to ITS-90, future revisions of the MeP will probably include improved approximations consistent with the best thermodynamic measurements. This may be managed by revising ITS-90 and PLTS-2000. 


\section{Proposals for the Wording of the New Definition}

An "explicit-unit definition" explicitly defines a unit in terms of a particular quantity of the same kind as the unit and, through a simple relationship implied by the definition itself or one or more laws of physics, implicitly fixes the value of a fundamental constant. Another option explicitly fixes the value of a fundamental constant and, through a simple relationship implied by the definition itself or one or more laws of physics, implicitly defines a unit; we call these "explicit-constant definitions." The explicit-unit definition of [17] for the kelvin is proposed as follows:

(1) The kelvin is the change of thermodynamic temperature that results in a change of thermal energy $k T$ by exactly $1.38065 X X \times 10^{-23}$ joule.

Here, and in the following proposals, the XX will be replaced with the appropriate digits of the Boltzmann constant when the new definition is established. The intention of the redefinition is to move away from any material substance/artifact and base the kelvin definition solely on a defined value for the Boltzmann constant. The new definition of the kelvin will be realized by a wide variety of primary thermometers. In the basic equations for all these thermometers appears the thermal energy $k T$ [18]. Definition (1) is simple and intuitive and would clearly endorse any appropriate method of measuring $k T$. However, the symbols $k$ and $T$ are undefined. Therefore, this slightly more complicated form is preferred:

(1a) The kelvin is the change of thermodynamic temperature $\boldsymbol{T}$ that results in a change of the thermal energy $k T$ by exactly $1.38065 \mathrm{XX} \times 10^{-23}$ joule, where $k$ is the Boltzmann constant.

All of the SI base units implicitly define measurement scales that have natural zeros and are therefore rational, i.e., all quantities can be expressed as ratios with respect to the base unit, e.g., $273.15 \mathrm{~K}=273.15 \times 1 \mathrm{~K}$. This is different on interval scales such as the Celsius scale where $100^{\circ} \mathrm{C}$ is not equal to $100 \times 1{ }^{\circ} \mathrm{C}$. On interval scales, the zero is arbitrary and the proportionality constant must be expressed in terms of the derivative. On a rational scale, it ought to be possible to express the proportionality constant absolutely. For this reason, "change of" in the following definition has been omitted. Moreover, a definition of the kelvin that relates to a gas is more easily understood, for example, by a high school student, than any other definition:

(2) The kelvin is the thermodynamic temperature at which the mean translational kinetic energy of atoms in an ideal gas at equilibrium is exactly (3/2) $\times 1.38065 \mathrm{XX} \times 10^{-23}$ joule.

Here, the broad but vague term "thermal energy" has been replaced by "mean translational kinetic energy." At the same time, this definition avoids questions about the kinetic energy associated with the internal degrees of freedom of a molecule by introducing clearly atoms as the particles under consideration. By using "atoms" in the plural, ensemble or time averages are included. However, the modifiers "ideal" and "equilibrium" are required if we are to remain accurate, even though modifiers interfere with clarity. This definition also includes the idea of an unbounded gas-but if we include zero-point energy, then "change of" must be there. Certainly, quantized systems need to include it, but an atom in an unbound (infinite volume) gas should have no zero-point energy. However, the biggest problem with definition (2) is that 
it is essentially a "material" (i.e., gas)-based definition rather than a principle-based definition.

To avoid the disturbing $3 / 2$ factor, the number of degrees of freedom, which is another complicating factor, has to be introduced:

(3) The kelvin is the thermodynamic temperature at which particles have an average energy of exactly $(1 / 2) \times 1.38065 X X \times 10^{-23}$ joule per accessible degree of freedom.

This definition does cover both time and ensemble averages easily but still has problems, most notably the zero-point energy. The question is whether one needs to define "particle" further-it cannot apply to all particles, e.g., photons, and how does it apply more generally to particles of any mass or spin? In this definition, we specify the number of degrees of freedom. This is the most general statement one can make.

Definitions 1-3 show how difficult it is to produce a satisfactory explicit-unit definition. Instead of being so specific, one could leave the definition sufficiently wide to encompass any form of primary thermometry and leave the mise en pratique to spell out the practical details. The explicit-constant definition in Mills et al. [17] for the kelvin follows this approach:

(4) The kelvin, unit of thermodynamic temperature, is such that the Boltzmann constant is exactly $1.38065 X X \times 10^{-23}$ joule per kelvin.

After thorough discussions, the TG-SI is recommending the explicit-constant definition (4) because it is sufficiently wide to accommodate future developments and does not favor any special primary thermometer for realizing the kelvin. Should the CCU decide to adopt explicit-unit definitions for the kilogram, the ampere, and the mole, then the second option of the TG-SI would be the formulation (1a) for the kelvin in order to be in line with the other new definitions.

\section{Progress of Experiments for Determination of the Boltzmann Constant}

The value of the molar gas constant, $R$, recommended by CODATA in 2002 [11] is essentially the weighted mean of two independent results for the speed of sound $u_{0}$ in argon obtained at a temperature close to and known in terms of the triple point of water, $T_{\text {TPW }}$. One result is from the National Institute of Standards and Technology (NIST), USA [19], with a relative uncertainty $u_{\mathrm{r}}=1.8 \times 10^{-6}$, and the other from the National Physical Laboratory (NPL), UK [20], with $u_{\mathrm{r}}=8.4 \times 10^{-6}$. Although the two results of acoustic gas thermometry (AGT) are consistent, because of the large difference in their uncertainties, the 2002 recommended value of $R$, and hence the 2002 recommended value of the Boltzmann constant $k$ with $u_{\mathrm{r}}(k)=1.8 \times 10^{-6}$, is to a very large extent determined by the NIST result.

In response to the recommendation $\mathrm{T} 2$ of the CCT [21], many projects have been started to measure independently the value of the Boltzmann constant. Methods based on the thermal equation of state of ideal gases are shown in Fig. 1, including their underlying basic equations [18]. These are, from left to right: the well-known constant-volume gas thermometry (CVGT), acoustic gas thermometry (as discussed in the previous paragraph and [22]), dielectric-constant gas thermometry (DCGT) using audio-frequency capacitance bridges [23], measurement of $n$ with refractive index gas 


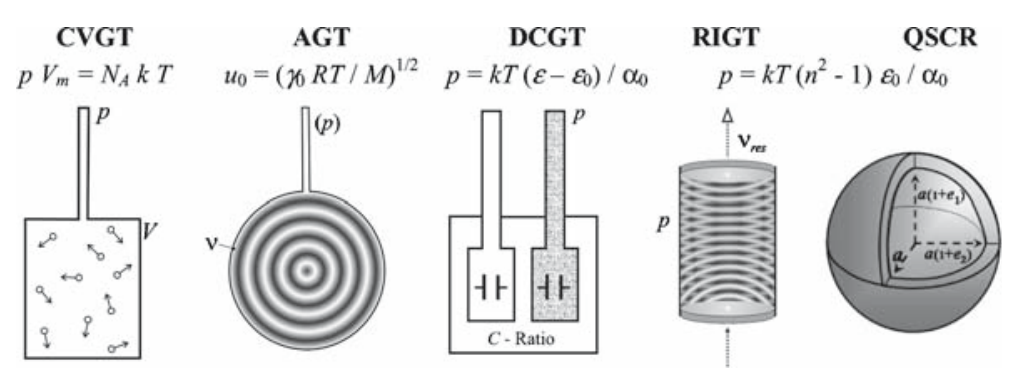

Fig. 1 Principles of constant-volume gas thermometry (CVGT), acoustic gas thermometry (AGT), dielectric-constant gas thermometry (DCGT), refractive index gas thermometry (RIGT), and thermometry using quasi-spherical cavity resonators (QSCR) $\left(\gamma_{0}=c_{p} / c_{V}\right.$ is the ratio of specific heat capacities at constant pressure and constant volume, $M$ is the molar mass, $\varepsilon_{0}$ is the dielectric constant, and $\alpha_{0}$ is the static electric dipole polarizations of the atom) [18]

Table 2 Uncertainty in determining the Boltzmann constant by applying different methods of primary thermometry [18]

\begin{tabular}{lcc}
\hline Method & Present state (ppm) & 2010 possibility (ppm) \\
\hline AGT & 2 & 1 \\
DCGT & 15 & 2 \\
TRT & 32 & 5 \\
QSCR & 40 & 10 \\
RIGT & 300 & 30 \\
DBT & 200 & 10 \\
\hline
\end{tabular}

thermometry (RIGT) applying optical resonators [24], and quasi-spherical cavity resonators (QSCRs) operating at gigahertz frequencies [25]. Other promising methods for determining $k$ are total radiation thermometry (TRT) [26,27], and Doppler-broadening thermometry (DBT) [28,29].

Table 2 gives a summary overview of the potential of the currently-available primary thermometers for determining the Boltzmann constant $k$, as deduced from the literature, a workshop held in 2005 at PTB [30], and recent information on new developments [18]. Table 2 illustrates that within the next four years there exists the possibility of achieving a reliable uncertainty of the value of $k$ of the order of one part in $10^{6}$ based on measurements applying different methods of primary thermometry. Thus, an improved value of the Boltzmann constant proposed for defining the kelvin would ideally have been determined by at least the two fundamentally different methods AGT and DCGT and be corroborated by other-preferably optical-measurements such as TRT and DBT with larger uncertainty.

The TG-SI appreciates the considerable progress of ongoing experiments to determine the Boltzmann constant in order to corroborate the present value. It is assumed that the experiments currently underway to measure $R$ or $k$ will achieve consistent results by the end of 2010, so that the CODATA group can recommend in its 2010 constants adjustment a new value for $k$ with a relative standard uncertainty about a factor of two smaller than the current $u_{\mathrm{r}}$ of approximately $2 \times 10^{-6}$. A value of $u_{\mathrm{r}}\left(T_{\mathrm{TPW}}\right)$ of about $1 \times 10^{-6}$, corresponding to about $0.25 \mathrm{mK}$, would reduce even more the 
insignificant differences between the thermodynamic uncertainties of columns 4 and 5 of Table 1 . The TG-SI will continue to monitor the progress of new determinations of the Boltzmann constant.

\section{Conclusions}

The Boltzmann constant is not connected with the other fundamental constants, in contrast to its macroscopic counterpart, the molar gas constant $R=k N_{\mathrm{A}}$. Thus, there are no alternatives to the linking of the kelvin aside from an exact value of the Boltzmann constant.

Our recommendation is that the unit kelvin of temperature $T$ should be defined by fixing the value of the Boltzmann constant, thus proceeding in the same way as with other units, with the aim to guarantee their long-term stability. The TG-SI is proposing that the value of the Boltzmann constant to be taken for the redefinition is as determined by the CODATA group in early 2011. For reasons of simplicity, our preference is for an explicit-constant definition with accompanying text explaining how the definition of the kelvin impacts upon primary and practical thermometry.

The new definition would be in line with modern science where nature is characterized by statistical thermodynamics, which implies the equivalence of energy and temperature as expressed by the Maxwell-Boltzmann equation $E=k T$ [31]. In principle, temperature could be derived from the measurement of energy. In practice, however, we have no simple and universal instrument for measuring energy and it appears in different forms, e.g., temperature. The fundamental constant $k$ converts the value of this measurable quantity into energy units.

Also, the Consultative Committee for Electricity and Magnetism (CCEM) established a corresponding working group to study proposed changes to the SI, in response to the recommendation of the CIPM [6]. The group organized a Round Table discussion on the proposed changes during the CPEM 2006 conference. Several experts from national metrology institutes, and academia as well, expressed a favorable opinion about fixing the Boltzmann constant to redefine the kelvin [32]. The experts in charge of studying the redefinition of the kilogram and the mole are also proposing to redefine the kelvin so that it is linked to an exactly defined value of the Boltzmann constant $[17,31]$.

It is not always necessary that a new definition of a SI base unit should immediately allow the unit to be realized with a reduced uncertainty [17]. The benefits to both metrology and science of replacing the current definition of the kelvin by one that links it to an exact value of the Boltzmann constant $k$, are viewed as outweighing any marginal increase in the uncertainty of thermodynamic temperature that might result. At very low and very high temperatures, there will be no need in the future to reference back to the triple point of water, which the TG-SI considers as the main practical advantage of the new definition.

As presented at the round-table discussion [32], the following time schedule for the adoption of the new definitions could be envisaged: If the required experimental data are available and in sufficient agreement in 2010, the values of the constants could be chosen based on the 2010 CODATA constants adjustment (31 December 2010 closing 
date). The new definitions could be adopted by the 24th CGPM in October 2011. Exact wordings of new definitions are to be developed by the CIPM through the CCU in collaboration with the Consultative Committees and other interested parties. Mises en pratique for the new unit definitions should be prepared by the CCM, CCEM, CCT, and CCQM, and available by 31 December 2011. The TG-SI understands that the updating of the mise en pratique for the definition of the kelvin [16] is under the responsibility of the special task group formed by CCT WG1. The president of the CCT will inform the other consultative committees of the work of this task group.

\section{References}

1. T.J. Quinn, Temperature, 2nd edn., Monographs in Physical Measurement (Academic Press, London, 1990)

2. H. Preston-Thomas, P. Bloembergen, T.J. Quinn, Supplementary Information for the International Temperature Scale of 1990 (BIPM, Sèvres, 1990)

3. H. Preston-Thomas, Metrologia 27, 3 (1990); ibid., p. 107

4. Appendix to Recommendation C1 (2000) Procès-Verbaux des Séances du Comité International des Poids et Mesures 68 (CIPM, Sèvres, 2001), pp. 129-130

5. J. Fischer, B. Fellmuth, Rep. Prog. Phys. 68, 1043 (2005)

6. Recommendation 1 (CI-2005): Preparative Steps Towards New Definitions of the Kilogram, the Ampere, the Kelvin and the Mole in Terms of Fundamental Constants (CIPM, Sèvres, 2005)

7. S. Chandrasekhar, An Introduction to the Study of Stellar Structure (Dover, New York, 1967), pp. 11-37

8. C.E. Shannon, AT\&T Tech. J. 27, 379 (1948); ibid., p. 623

9. M. Planck, Theorie der Wärmestrahlung, 4th edn. (Barth, Leipzig, 1921)

10. D.R. White, M. Ballico, D. del Campo, S. Duris, E. Filipe, A. Ivanova, A. Kartal Dogan, E. MendezLango, C. Meyer, F. Pavese, A. Peruzzi, E. Renaot, S. Rudtsch, K. Yamazawa, in Proceedings of TEMPMEKO 2007 (to be published in Int. J. Thermophys.)

11. P.J. Mohr, B.N. Taylor, Rev. Mod. Phys. 77, 1 (2005)

12. IEC 60584-2, Int. Electrotech. Comm., Geneva, www.iec.ch (1989)

13. IEC 60751, Int. Electrotech. Comm., Geneva, www.iec.ch (1995)

14. D.C. Ripple, K.S. Gam, Y. Hermier, K.D. Hill, R.L. Rusby, A.G. Steele, P.P.M. Steur, M. Stock, G.F. Strouse, D.R. White, Report of the Ad Hoc Task Group on the Triple Point of Water, Working Documents of the 23rd Meeting of the Consultative Committee for Thermometry (BIPM, Document CCT/05-07, 2005)

15. Recommendation 2 (CI-2005): Clarification of the Definition of the Kelvin, Unit of Thermodynamic Temperature (CIPM, Sèvres, 2005)

16. Mise en Pratique for the Definition of the Kelvin, adopted by the CCT in April 2006 (BIPM, http:// www.bipm.org/utils/en/pdf/MeP_K.pdf, 2006)

17. I.M. Mills, P.J. Mohr, T.J. Quinn, B.N. Taylor, E.R Williams, Metrologia 43, 227 (2006)

18. B. Fellmuth, Ch. Gaiser, J. Fischer, Meas. Sci. Technol. 17, R145 (2006)

19. M.R. Moldover, J.P.M. Trusler, T.J. Edwards, J.B. Mehl, R.S. Davis, J. Res. Natl. Bur. Stand. (U.S.) 93, 85 (1988)

20. A.R. Colclough, T.J. Quinn, T.R.D. Chandler, Proc. R. Soc. London A 368, 125 (1979)

21. Recommendation T 2 (2005) to the CIPM: New Determinations of Thermodynamic Temperature and the Boltzmann Constant. Working Documents of the 23rd Meeting of the Consultative Committee for Thermometry (BIPM, Document CCT/05-31, 2005)

22. R.M. Gavioso, P.A. Giuliano Albo, G. Benedetto, R. Spagnolo, in Proceedings of CPEM 2006 (CPEM, Turin, 2006), pp. 26-27

23. B. Fellmuth, J. Fischer, C. Gaiser, N. Haft, in Proceedings of TEMPMEKO 2004, 9th International Symposium on Temperature and Thermal Measurements in Industry and Science, Vol. 2, ed. by D. Zvizdić, L.G. Bermanec, T Stašić, T. Veliki (LPM/FSB, Zagreb, Croatia, 2005), pp. 73-78

24. J.A. Stone, A. Stejskal, Metrologia 41, 189 (2004)

25. E.F. May, L. Pitre, J.B. Mehl, M.R. Moldover, J.W. Schmidt, Rev. Sci. Instrum. 75, 3307 (2004) 
26. T.J. Quinn, J.E. Martin, Phil. Trans. R. Soc. A 316, 85 (1985)

27. J.E. Martin, P.R. Haycocks, Metrologia 35, 229 (1998)

28. C.J. Bordé, Phil. Trans. R. Soc. A 363, 2177 (2005)

29. C. Daussy, M. Guinet, A. Amy-Klein, K. Djerroud, Y. Hermier, S. Briaudeau, Ch.J. Bordé, C. Chardonnet, arXiv/quant-ph/0701176 and Phys. Rev. Lett. 98, 250801 (2007)

30. B. Fellmuth, J. Fischer, C. Gaiser, W. Buck, Workshop on Methods for New Determinations of the Boltzmann Constant, Working Documents of the 23rd Meeting of the Consultative Committee for Thermometry (BIPM, Document CCT/05-02, 2005)

31. P. Becker, P. De Bièvre, K. Fujii, M. Glaeser, B. Inglis, H. Luebbig, G. Mana, Metrologia 44, 1 (2007)

32. M. Stock, T. Witt, Metrologia 43, 583 (2006) 